\title{
Circadian Plasma Cortisol Measurements Reflect Severity of Hypercortisolemia in Children with Different Etiologies of Endogenous Cushing Syndrome
}

\author{
Amit Tirosha $^{\mathrm{a} b}$ Maya B. Lodish ${ }^{\mathrm{a}}$ Charalampos Lyssikatos $^{\mathrm{a}}$ Elena Belyavskaya ${ }^{\mathrm{a}}$ \\ Georgios Z. Papadakis ${ }^{a, c}$ Constantine A. Stratakis ${ }^{\mathrm{a}}$ \\ a Section on Endocrinology and Genetics, Eunice Kennedy Shriver National Institute of Child Health and Human \\ Development, National Institutes of Health, Bethesda, MD, USA; 'b Sackler Faculty of Medicine, Tel Aviv University, \\ Tel Aviv, Israel; ' $R$ Radiology and Imaging Sciences, Warren Grant Magnuson Clinical Center, National Institutes of \\ Health, Bethesda, MD, USA
}

\section{Keywords}

Hypercortisolemia; cushing · Circadian cortisol

\begin{abstract}
Background: The utility of circadian cortisol variation in estimating the degree of hypercortisolemia in different forms of endogenous Cushing syndrome (CS) has not been evaluated in children yet. Methods: A retrospective cohort study, including children who underwent surgery due to CS ( $n=$ $115)$, was divided into children with a pituitary adenoma (Cushing disease) $(n=88)$, primary adrenal CS $(n=21)$, or ectopic adrenocorticotropin- or corticotropin-releasing hormone (ACTH-/CRH)-secreting tumors $(n=6)$. Circadian plasma cortisol measurements were obtained at 11:30 p.m. and at midnight, and at 7:30 and 8:00 a.m. The ratios between the morning and late-night concentrations were calculated. Results: Plasma cortisol early-morning and midnight (AM/PM) ratios negatively correlated with 24-h urinary free cortisol (UFC) collections among the full study population and in each of the individual etiologies. Plasma ACTH concentrations positively correlated with plasma cor-
\end{abstract}

\section{KARGER}

(c) 2017 S. Karger AG, Basel

E-Mail karger@karger.com

www.karger.com/hrp tisol AM/PM ratios among patients with ACTH-independent CS. Finally, patients with primary pigmented nodular adrenocortical disease showed no correlation between UFC collections and the plasma cortisol AM/PM ratio, in contrast with other etiologies for primary adrenal CS, which showed a strong negative correlation between them. Conclusion: Our study shows the association between the plasma cortisol AM/PM ratio and the degree of hypercortisolemia in children with CS.

(C) 2017 S. Karger AG, Basel

\section{Introduction}

Endogenous Cushing syndrome (CS) is caused by excess secretion of adrenocorticotropin (ACTH) or cortisol leading to dysregulation of the hypothalamic-pituitaryadrenal (HPA) axis. Thus, primary adrenal CS is due to autonomous secretion of cortisol from the adrenal gland; Cushing disease (CD) is caused by an ACTH-secreting pituitary adenoma, and ectopic CS is caused by ACTH and/or CRH secretion from a nonpituitary neoplasm [1]. 
Table 1. Patients' demographic and baseline biochemical characteristics according to patients' diagnoses

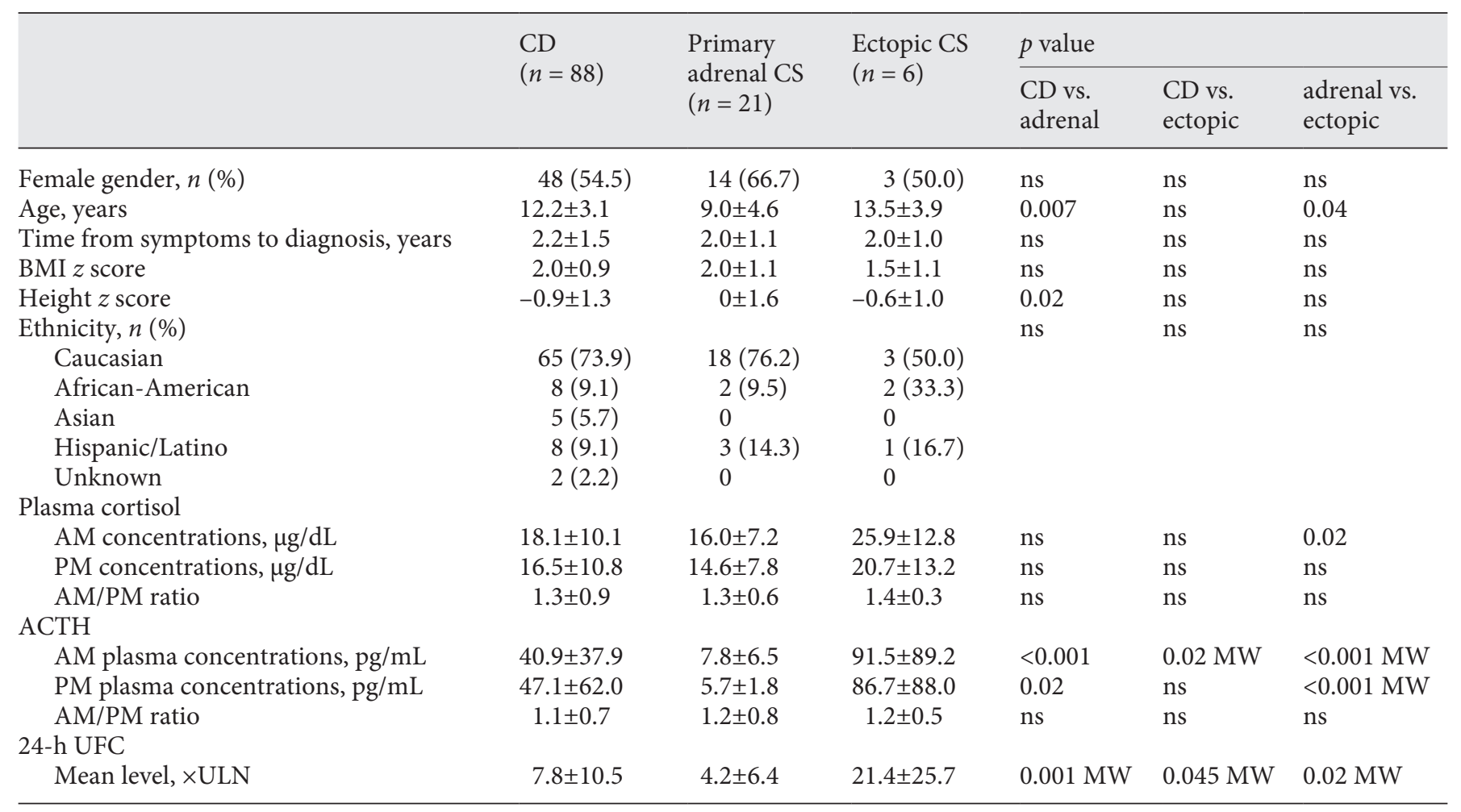

Data are presented as mean \pm standard deviation unless mentioned otherwise. CD, Cushing disease; CS, Cushing syndrome; BMI, body mass index; ACTH, adrenocorticotropin hormone; MW, Mann-Whitney U test; UFC, urinary free cortisol; ULN, upper limit of the reference range. Comparisons were performed using ANOVA and $\chi^{2}$ tests for continuous and categorical variables, respectively.

The normal physiology of cortisol secretion follows a circadian pattern, peaking after awakening in the morning and reaching a nadir around midnight [2]. However, among adult patients with CS, circadian cortisol variation is diminished [3]. Hence, the current guidelines for CS diagnosis in adult patients cite late-night hypercortisolemia as measured by midnight salivary cortisol as a criterion for CS diagnosis [4], and similar tests are used for CS diagnosis in children [5]. Moreover, beyond its use for CS diagnosis, diminished circadian cortisol variation is both a cardiovascular [6] and metabolic risk factor [7], even among normocortisolemic subjects.

In the current analysis, we investigate the circadian cortisol variation among children with different etiologies of CS, as expressed by the ratio between early-morning and midnight (AM/PM) plasma cortisol. We analyze the association between this variation and 24-h urinary free cortisol (UFC) collections among patients with different etiologies for CS.

\section{Patients and Methods}

We conducted a retrospective cohort study including consecutive patients aged $\leq 18$ years who were admitted to the National Institutes of Health for evaluation of suspected CS between 1995 and 2015. All patients were recruited through clinical protocols 97-CH-0076, 95-CH-0059, and 00-CH-0160 conducted by the Eunice Kennedy Shriver National Institute of Child Health and $\mathrm{Hu}$ man Development (NICHD), and their families gave written informed consent.

All patients were hospitalized and underwent screening tests for CS, including serial 24-h UFC collections and circadian plasma cortisol measurements. Circadian plasma cortisol measurements were performed during hospitalization, using an indwelling venous catheter with designated extension to avoid sleep disturbance during the night measurements. Blood samples were drawn at 7 : 30 and 8:00 a.m. for the morning measurements and at 11:30 p.m. and midnight for the late-night samples ( 2 measurements in the morning and 2 at late night). Calculated mean values of the a.m. and p.m. samples were used for the final analysis. The current analysis included patients $\leq 18$ years with either elevated 24 -h UFC or elevated midnight plasma cortisol $(>4.4 \mu \mathrm{g} / \mathrm{dL})$. Patients diagnosed with CS underwent further investigation in order to locate 


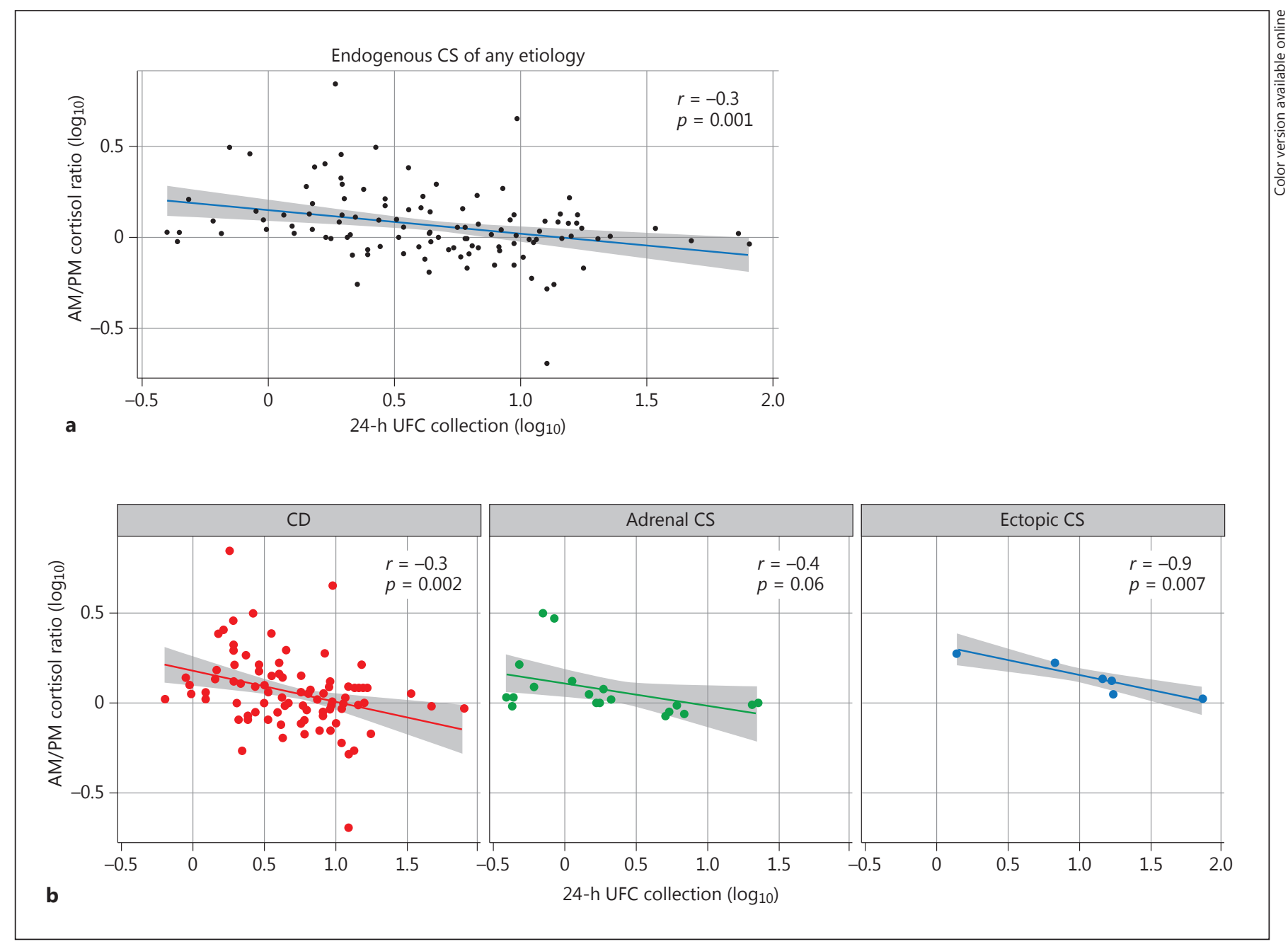

Fig. 1. Correlation analysis between 24-h UFC collection and the plasma cortisol AM/PM ratio in the entire cohort (a) and according to the patient's CS etiology (b). UFC, urinary free cortisol; CS, Cushing syndrome; AM/ PM, early-morning/midnight.

the source of hypercortisolemia. Patients then went on to surgical intervention, either adrenalectomy or transsphenoidal surgery, as appropriate.

All pathological reports were reviewed by the authors; CD was defined as a pituitary adenoma with positive ACTH immunohistochemistry, and adrenal lesions were defined as previously described [8].

\section{Statistical Analysis}

Statistical calculations were performed with SPSS 20.0 software (SPSS Inc., Chicago, IL, USA) and $R$ statistics, version 3.2.2. Results are expressed as mean \pm standard deviation (SD) unless otherwise indicated. For group comparisons, the independent Student's $t$ test or one-way analysis of variance (ANOVA) were used to analyze differences in numerical variables, and the $\chi^{2}$ test was employed to analyze differences in categorical variables. Variables with a non-normal distribution were analyzed using nonparamet-

Circadian Plasma Cortisol Measurements in Cushing ric tests, as appropriate. The Pearson product was used for analysis of correlations between variables. The $p$ value for significance was set at $<0.05$.

\section{Results}

\section{Description of the Cohort and Overall Data}

The current analysis included 115 children, 65 females $(56.5 \%)$, aged $11.7 \pm 3.7$ years. The patients' mean time from the first symptom to diagnosis was $2.1 \pm 1.4$ years. Eighty-eight patients (76.5\%) had CD, 21 (18.3\%) had primary adrenal CS (3 adrenal adenomas, 7 micronodular hyperplasia, 1 macronodular hyperplasia, and 


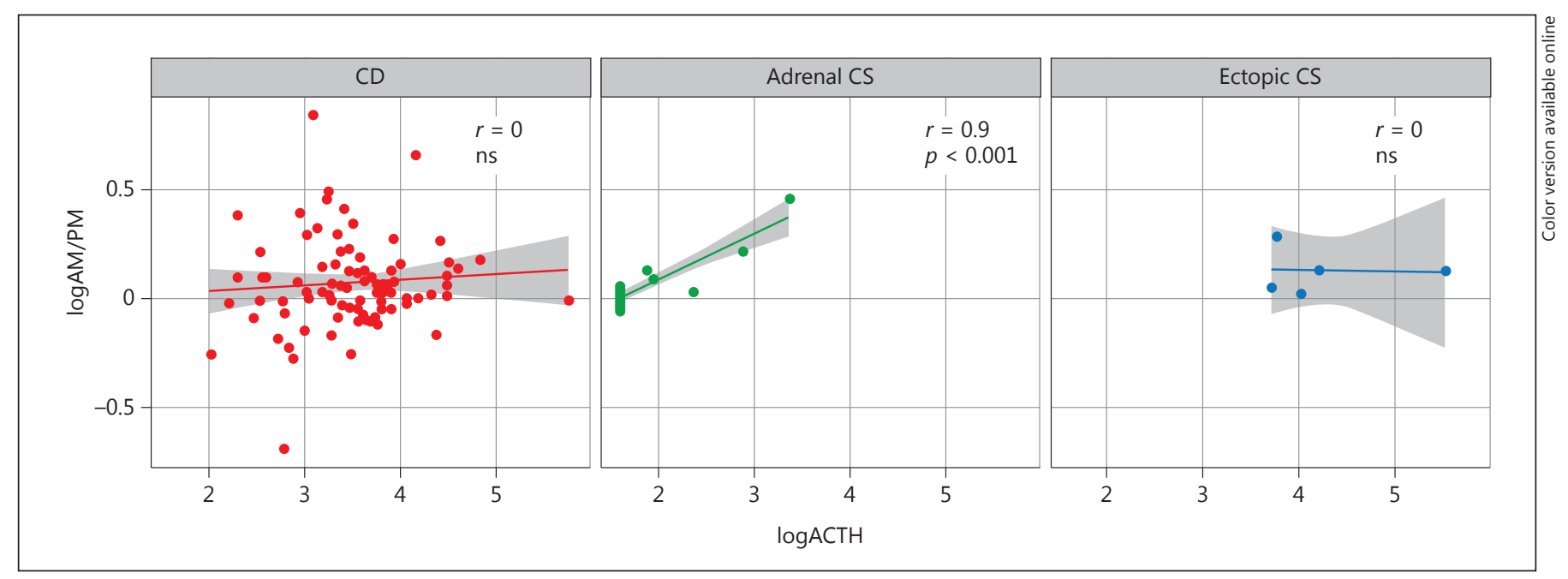

Fig. 2. Correlation analysis between morning plasma ACTH concentrations and the plasma cortisol AM/PM ratio according to the etiology of CS. CS, Cushing syndrome; ACTH, adrenocorticotropin hormone; CS, Cushing syndrome; AM/PM, early-morning/midnight.

10 primary pigmented nodular adrenocortical disease [PPNAD]), and 6 patients (5.2\%) had ectopic ACTH-/ $\mathrm{CRH}$-secreting tumors.

Mean plasma cortisol concentrations for the whole cohort were $16.4 \pm 10.4 \mu \mathrm{g} / \mathrm{dL}$ at midnight and $18.2 \pm$ $9.9 \mu \mathrm{g} / \mathrm{dL}$ when measured in the morning, with an AM/ PM ratio of $1.3 \pm 0.8$ and a mean 24 -h UFC of $7.9 \pm 11.6$ times the upper limit of the reference range $(\times \mathrm{ULN})$. The patients' mean BMI $z$ score was $1.9 \pm 0.9$, and the mean height $z$ score was $-0.7 \pm 1.3$. Patients with ectopic CS had the highest and those with primary adrenal CS had the lowest 24-h UFC collections. Demographic and biochemical characteristics of the patients according to their histopathological diagnoses are detailed in Table 1.

\section{UFC and AM/PM Cortisol Ratio Correlation in the Different Etiologies of CS}

Patients with lower 24-h UFC collections $(<2 \times U L N$, $n=28$ ) retained circadian cortisol rhythmicity more than those having higher 24-h UFC collections $(n=82,1.8 \pm$ 1.2 vs. $1.1 \pm 0.6 \times \mathrm{ULN}, p=0.01$, respectively). This was also reflected in the negative correlation between 24 -h UFC collections and plasma cortisol AM/PM ratios ( $n=$ $111, r=-0.3, p=0.001)$. Moreover, a subgroup analysis according to the CS etiology revealed a weak negative correlation among patients with $\mathrm{CD}(r=-0.3, p=0.002)$, a similar trend among those with primary adrenal CS $(r=-0.4, p=0.06)$, and a strong negative correlation among subjects with CS due to ACTH-/CRH-secreting tumors ( $r=-0.9, p=0.007$, Fig. 1$)$.

Morning ACTH concentrations positively correlated with the plasma cortisol $\mathrm{AM} / \mathrm{PM}$ ratio among patients with primary adrenal CS $(r=0.9, p<0.001)$ but had no correlation $(r=0)$ among those with either CD or ectopic ACTH-/CRH-secreting tumors (Fig. 2).

\section{Primary Adrenal CS: Comparison between Different Etiologies}

Patients with PPNAD had comparable mean plasma cortisol AM/PM ratios ( $1.3 \pm 0.7$ vs. $1.2 \pm 0.6, \mathrm{~ns})$, mean 24-h UFC collections ( $3.6 \pm 6.9$ vs. $4.9 \pm 6.2 \times \mathrm{ULN}, \mathrm{ns})$, mean midnight $(12.1 \pm 6.4$ vs. $16.9 \pm 8.5 \mu \mathrm{g} / \mathrm{dL}$, ns $)$ and morning plasma cortisol concentrations ( $14.2 \pm 6.2$ vs. 17.6 $\pm 7.9 \mu \mathrm{g} / \mathrm{dL}$, ns), and mean morning plasma ACTH concentrations ( $7.6 \pm 4.7$ vs. $7.9 \pm 8.1$, ns) with subjects having other etiologies of primary adrenal CS. In addition, a strong negative correlation $(r=-0.8, p=0.01)$ was found among those with other etiologies for primary adrenal CS (Fig. 3).

\section{Discussion}

In the current analysis, we investigated the association between the plasma cortisol AM/PM ratio and 24-h UFC values in children with different etiologies of CS. We found that patients with a higher degree of cortisolemia had more loss of circadian cortisol rhythmicity (Fig. 1), as 
Fig. 3. Difference in circadian cortisol variation between patients with PPNAD versus other primary adrenal CS. Mean plasma cortisol $\mathrm{AM} / \mathrm{PM}$ ratio and the correlation between 24-h UFC collection and the plasma cortisol AM/PM ratio. UFC, urinary free cortisol; PPNAD, primary pigmented nodular adrenocortical disease; AM/PM, early-morning/midnight.

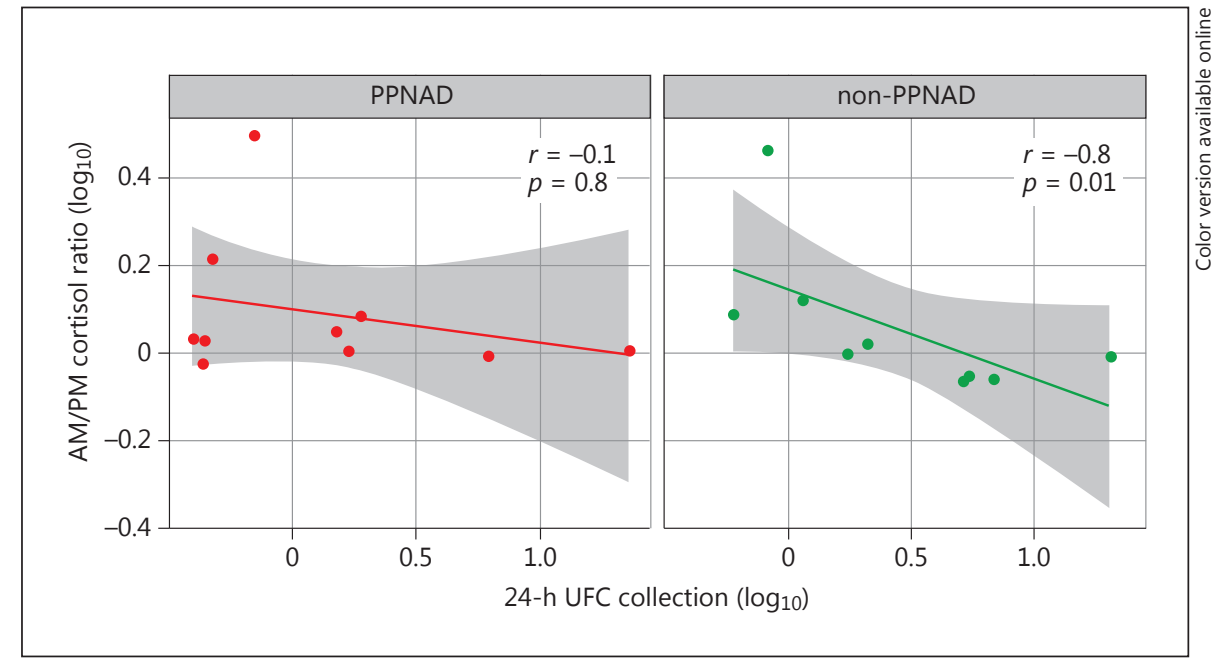

expected. We found a strong positive correlation between ACTH concentrations and the plasma cortisol AM/PM ratio among patients with primary adrenal CS, whereas no correlation was found among subjects with either CD or ectopic ACTH-/CRH-secreting tumors (Fig. 2). Finally, among patients with primary adrenal CS, subjects with a primary adrenal CS other than PPNAD showed a strong negative correlation between these 2 measures (Fig. 3 ).

Measurement of midnight plasma cortisol in adults was found to be highly sensitive for diagnosing CS [9]. Similarly, a late-night plasma cortisol measurement of $<4.4 \mu \mathrm{g} / \mathrm{dL}$ in children had $100 \%$ specificity for ruling out CS [5]. We used the plasma cortisol AM/PM ratio as an estimate for circadian variation and showed that it negatively correlated with 24-h UFC collections, a valid measure for hypercortisolemia, among all etiologies for CS (Fig. 1).

Suppression of plasma ACTH concentrations due to elevated plasma cortisol concentrations is expected in a normally functioning HPA axis [10]. In patients with ACTH- or CRH-secreting tumors, plasma ACTH concentrations mainly reflect the degree of the tumor's secretion, whereas among those with autonomous adrenal cortisol, the pituitary ACTH secretion is low due to negative feedback. Thus, it is not surprising to find lack of correlation between plasma ACTH concentrations and plasma $\mathrm{AM} / \mathrm{PM}$ ratios among subjects with $\mathrm{CD}$ or ectopic CS, and a preserved correlation among those with primary adrenal CS (Fig. 2), who obviously did not have a fully suppressed HPA axis.

PPNAD is caused by a germline mutation in the gene encoding the regulatory subunit of protein kinase $\mathrm{A}$ (PRKAR1A) [11], and usually presents as part of the Carney

Circadian Plasma Cortisol Measurements in Cushing complex [12]. We have shown lack of correlation between 24-h UFC collections and circadian cortisol rhythmicity in PPNAD (Fig. 3), in contrast to a high correlation between them in other forms of primary adrenal CS. This is in light of comparable plasma and urine cortisol concentrations between PPNAD and non-PPNAD primary adrenal CS. We suggest 3 possible explanations for the lack of circadian cortisol rhythmicity in PPNAD patients compared with other primary adrenal CS etiologies. First, the lack of unaffected adrenocortical cells in the PPNAD-affected adrenal cortex, compared with other etiologies for primary adrenal $\mathrm{CS}$, means that there are few, if any, ACTH-responsive cells that produce cortisol. Second, there might be a higher endogenous cortisol production activity of the PPNAD-affected adrenocortical cells compared with other etiologies. Third, adrenocortical cells in PPNAD might have an altered expression of "clock genes" [13] that have been demonstrated in unaffected adrenocortical cells.

The main strength of this study is the large sample size as well as the full and uniform evaluation performed for all patients. Moreover, all diagnoses for the study subjects were classified according to the histopathological evaluation.

In conclusion, we have shown that a lower circadian cortisol rhythmicity reflects a higher degree of cortisolemia in general, and specifically in various forms of endogenous CS. It is particularly useful in identifying patients with PPNAD and distinguishing them from those with other forms of ACTH-dependent cortisol secretion.

\section{Disclosure Statement}

The authors have nothing to disclose. 


\section{References}

$>1$ Lacroix A, Feelders RA, Stratakis CA, Nieman LK: Cushing's syndrome. Lancet 2015;386: 913-927.

-2 Glass AR, Zavadil AP, Halberg F, Cornelissen G, Schaaf M: Circadian rhythm of serum cortisol in Cushing's disease. J Clin Endocrinol Metab 1984;59:161-165.

$\checkmark 3$ Lodish MB, Trivellin G, Stratakis CA: Pituitary gigantism: update on molecular biology and management. Curr Opin Endocrinol Diabetes Obes 2016;23:72-80.

-4 Nieman L, Biller B, Findling J, Newell-Price J, Savage M, Stewart P, et al: The diagnosis of Cushing's syndrome: an Endocrine Society Clinical Practice Guideline. J Clin Endocrinol Metab 2008;95:1526-1540.

5 Batista DL, Riar J, Keil M, Stratakis CA: Diagnostic tests for children who are referred for the investigation of Cushing syndrome. Pediatrics 2007;120:e575-e586.
6 Ronaldson A, Kidd T, Poole L, Leigh E, Jahangiri M, Steptoe A: Diurnal cortisol rhythm is associated with adverse cardiac events and mortality in coronary artery bypass patients. J Clin Endocrinol Metab 2015;100:3676-3682.

7 Hackett RA, Kivimäki M, Kumari M, Steptoe A: Diurnal cortisol patterns, future diabetes, and impaired glucose metabolism in the Whitehall II Cohort Study. J Clin Endocrinol Metab 2016;101:619-625.

8 Stratakis CA: Cushing syndrome caused by adrenocortical tumors and hyperplasias (corticotropin-independent Cushing syndrome) Endocr Dev 2008;13:117-132.

9 Newell-Price J, Trainer P, Perry L, Wass J, Grossman A, Besser M: A single sleeping midnight cortisol has $100 \%$ sensitivity for the diagnosis of Cushing's syndrome. Clin Endocrinol (Oxf) 1995;43:545-550.
0 Keller-Wood ME, Dallman MF: Corticosteroid inhibition of ACTH secretion. Endocr Rev 1984;5:1-24.

11 Kirschner LS, Carney JA, Pack SD, Taymans SE, Giatzakis C, Cho YS, et al: Mutations of the gene encoding the protein kinase A type I-alpha regulatory subunit in patients with the Carney complex. Nat Genet 2000;26:89-92.

$>12$ Almeida MQ, Stratakis CA: Carney complex and other conditions associated with micronodular adrenal hyperplasias. Best Pract Res Clin Endocrinol Metab 2010;24:907914

13 Valenzuela FJ, Torres-Farfan C, Richter HG, Mendez N, Campino C, Torrealba F, et al: Clock gene expression in adult primate suprachiasmatic nuclei and adrenal: is the adrenal a peripheral clock responsive to melatonin? Endocrinology 2008;149:1454-1461. 